(c) 2018 IEEE. Personal use of this material is permitted. Permission from IEEE must be obtained for all other uses, in any current or future media, including reprinting/republishing this material for advertising or promotional purposes, creating new collective works, for resale or redistribution to servers or lists, or reuse of any copyrighted component of this work in other works.

Digital Object Identifier (DOI): 10.1109/TIE.2017.2733450

IEEE Transaction on Industrial Electronics (Volume: 65, Issue: 2, Jan. 2018)

Analysis and Stabilization of a Smart Transformer-Fed Grid

Zhi-Xiang Zou

Giampaolo Buticchi

Marco Liserre

Suggested Citation

Z. X. Zou, G. Buticchi and M. Liserre, "Analysis and Stabilization of a Smart Transformer-Fed Grid," IEEE Transactions on Industrial Electronics, vol. 65, no. 2, pp. 1325-1335, Feb. 2018. 


\title{
Analysis and Stabilization of a Smart Transformer-Fed Grid
}

\author{
Zhi-Xiang Zou, Student Member, IEEE, Giampaolo Buticchi, Senior Member, IEEE, \\ and Marco Liserre, Fellow Member, IEEE
}

\begin{abstract}
The stability of the grid-connected inverters adopted in Distributed Energy Resources (DERs) highly depends on the characteristics of the grid impedance. Hence, various active damping methods and adaptive control algorithms have been proposed for the control of the grid inverters. Differently from those existing solutions, this paper proposes to use a Smart Transformer (ST) to shape the grid impedance to interact with the controllers of local inverters, aiming at improving the stability of the local controllers as well as the overall grid. A main advantage of this solution is that it offers a pervasive service to all the available DERs, reducing the cost and design effort of the local controllers. Three active damping methods are presented in this paper and the implementation issues in the ST LV side voltage controller are given. Moreover, the design criteria of the active damping methods considering the control performance of ST and the stability requirements of local DERs are presented. Analysis and experiments are carried out to verify the effectiveness of the proposed solution.
\end{abstract}

Index Terms-Solid state transformer, smart transformer, distribution grid, stability, active damping.

\section{INTRODUCTION}

$\mathbf{T}$ HE penetration of Distributed Energy Resources (DERs) is growing rapidly in distribution grids and microgrids. Being affected by the control dynamics and switching techniques, the stability problems arise which can be studied by small-signal analysis [1], [2]. In the context, the stability of an electric grid dominated by the grid inverters have drawn wide attentions, particularly focusing on the topics including system modeling [3], control and filter design [4], stability assessment [5], etc.

One important aspect determining the stability of grid inverters is the ratio of grid impedance to inverter impedance [3], [6]. Once the inverter impedance is fixed, the variation of grid impedance would incur stability issues and lead to the performance degradation of the overall grid. With this consideration, in a traditional distribution grid, one possibility is to design an appropriate inverter impedance which can adapt to a certain range of grid impedance variation [7]-[9]. On

Manuscript received Feb. 01, 2017; revised Mar. 30 and May 24, 2017; accepted Jun. 08, 2017. This work was supported in part by the European Research Council under the European Union's Seventh Framework Programme (FP/2007-2013) / ERC Grant Agreement n. [616344] - HEART.

Zhi-Xiang Zou, G. Buticchi and M. Liserre are with the Chair of Power Electronics, Christian-Albrechts University of Kiel, Kiel, Germany, (email: zz, gibu, ml@tf.uni-kiel.de). the other hand, the newly proposed Smart Transformer (ST) concept provides another possibility to mitigate the stability issues of the grid inverters [10]. The ST is a solid-state transformer adopted as intelligent substation with control and communication functionalities. One scenario is to adapt the dynamic properties of the ST in order to interact with the grid inverters of DERs. Like the active dampers [11], which can reshape the equivalent grid impedance to the paralleled grid inverters at same PCC, the idea is to directly reshape the grid impedance by means of the ST that is connected to the LV AC grid in series, aiming at modifying the relationship between grid impedance and inverter impedance so that the ST can facilitate the stabilization of all local grid inverters as well as the whole grid. Compared to the traditional decentralized stabilization solutions, the reshaping of the ST output impedance is expected to be an indispensable service provided to all the available DERs and inverter-interfaced loads with "plug-andplay" functionality. It can avoid complex control/computation and measurements being implemented locally, and therefore reduces the cost of the DERs. Rather than designing a robust local control system, the ST can rapidly adjust the grid profiles to meet the evolving and unpredictable requirements from utility and local customers.

In general, the objective of this paper is to propose a centralized stabilization scheme by means of ST to address instability caused by inverter-interfaced DERs. To show the superiority of the proposed scheme, this paper begins with a comparison between a traditional grid and a ST-fed grid and their stability analysis are given by using Nyquist stability criterion. To support the proposed schemes, three active damping methods are presented and implemented in the ST LV side voltage controller to interact with the grid inverters so that the potential stability problems could be solved. To study the effectiveness of the proposed schemes and active damping methods, both experimental verification and benchmark grid evaluation by using a Real Time Power System (RTDS) simulator have been carried out. It is known that the Flexible Alternating Current Transmission System (FACTS) is one of the most efficient alternatives in transmission system against power system problems [12], while using a ST in power grids makes it possible to facilitate system operation in MV/LV distribution gird. The paper structure is organized as follows: The stability issues of the traditional transformer-fed grid and the ST-fed grid are given in Section II. In order to flexibly stabilize the grid, in Section III three active damping methods are presented and implemented in the ST, aiming at reshaping the 


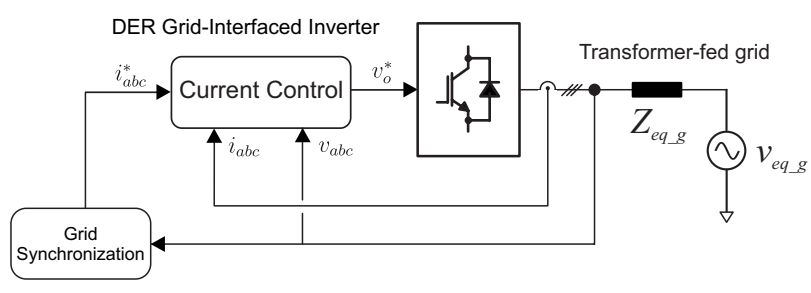

(a)

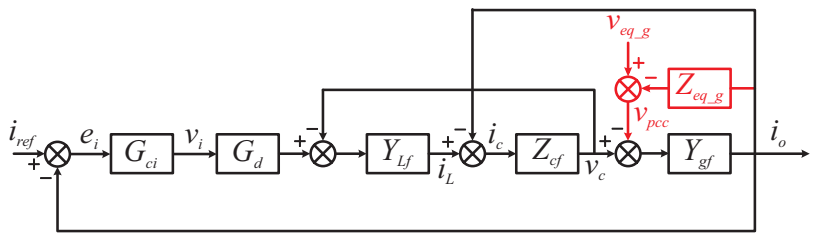

(b)

Fig. 1. Grid integration of a DER inverter in a traditional transformer-fed grid: (a) system configuration, and (b) schematic diagram of a currentcontrolled grid inverter.

impedance of the ST so that the holistic stability margin of the ST-fed grid is improved. Comprehensive design criteria of the proposed solutions are demonstrated in Section IV. Experimental and RTDS results are provided in Section V to verify the effectiveness of the proposed solutions. Conclusions are drawn in Section VI.

\section{StABILITY ISSUES IN LV DISTRIBUTION GRIDS}

This section describes the stability issues of grid-connected inverters in a traditional power transformer-fed grid and a STfed grid.

\section{A. Stability Issue of a Traditional Transformer-fed Grid}

In a traditional transformer-fed grid, the DERs are controlled as a constant power/current source and the grid can be represented by a Thévenin circuit (see Fig. 1a). The grid inverter is connected to the Point of Common Coupling (PCC) through an output filter, and a synchronization and a power/current controller are necessary to regulate the output power/current of the DER. To investigate the influence of the grid impedance on the grid inverter, a schematic diagram of a current-controlled grid inverter with an $L C L$ filter considering the grid impedance is shown in Fig. 1b. The grid impedance $Z_{e q_{-} g}$ is coupled to the closed-loop system. $G_{c i}$ is the transfer function of the current controller, $G_{d}$ represents a one-andhalf-sample computational and PWM delay, $Y_{L f}=1 / L_{f f} s$, $Y_{g f}=1 / L_{g f} s, Z_{c f}=1 / C_{f} s$ are the admittance of the inverter-side inductor and the grid-side inductor, and the impedance of the capacitor, of the $L C L$ filter, respectively. Based on Fig. 1b, the open-loop transfer function is

$$
\begin{aligned}
G_{o p_{\_} d e r}(s) & =G_{c i}(s) G_{d}(s) Z_{c f}(s) Y_{L f}(s) Y_{g f}(s) \\
& \cdot\left[Z_{c f}(s)\left(Y_{L f}(s)+Y_{g f}(s)\right)+1\right. \\
& \left.+Y_{g f}(s) Z_{e q \_g}(s)\left(1+Z_{c f}(s) Y_{L f}(s)\right)\right]^{-1} .
\end{aligned}
$$

In practical cases, $Z_{e q \_g}$ would vary in a certain range depending on the grid configuration in terms of cable length,

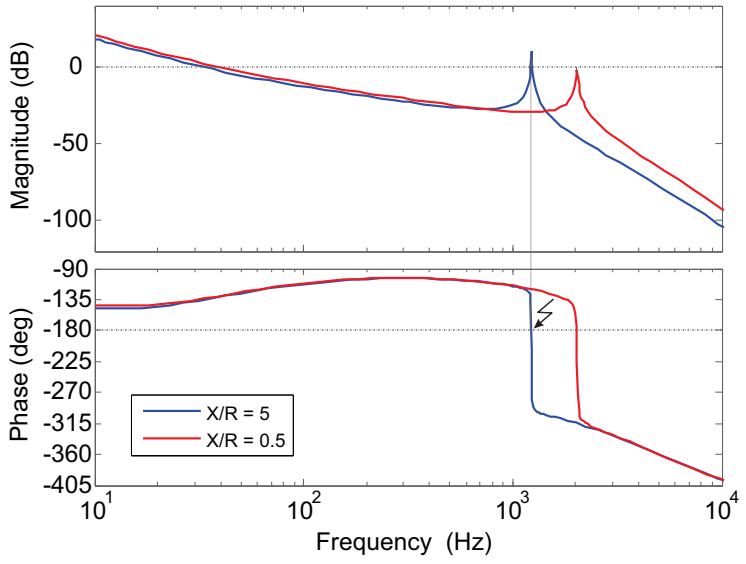

Fig. 2. Open-loop bode diagrams for DER inverter connected to a grid with different grid impedance.

power transformer parameter, and loads as well as "plug-andplay" devices [13]. Thus the stability margin of (1) would change. An example of the stability of grid inverter under different grid impedance conditions is studied and the corresponding Bode diagram is shown in Fig. 2. In this case, a Proportional Integral (PI) controller is employed as the current controller, an $L C L$ output filter with the parameters of $L_{f f}=5.03 \mathrm{mH}, L_{g f}=0.5 \mathrm{mH}$, and $C_{f}=10 \mu \mathrm{F}$ is used, and two different grid impedance are considered. Initially, the current control system considering $Z_{e q_{-} g}=0.00025 \cdot s+0.15$ $(\mathrm{X} / \mathrm{R}$ ratio is 0.5$)$ is designed with a phase margin of $49.5 \mathrm{deg}$ (red curve in Fig. 2). When the grid impedance changes, the stability of the control system can not always be guaranteed. From the Bode diagram, it is seen that a $-180^{\circ}$ crossing occurs at the neighborhood of the resonant frequency where the magnitude is positive, when a different grid impedance is considered, for example, $\mathrm{X} / \mathrm{R}$ ratio is 5 , shown by the blue curve in Fig. 2. As a result, the stability of a gridconnected inverter cannot always be guaranteed in a traditional transformer-fed grid even it is designed with an acceptable stability margin. To address this issue, advanced control algorithms for DER inverters have been proposed in literature, aiming at better adapting to various grid conditions. The mathematical expressions of the interaction between the DER impedance and the grid impedance was firstly presented in [7], giving clear guidelines of inverter impedance shaping in certain frequency range. Further work pointed out that by using active/passive damping methods [8], the grid inverter impedance can be optimized, largely avoiding instability. Moreover, adaptive control is another strong candidate to shape online the inverter impedance based on the real conditions of the grid impedance,. For example, in [9], an adaptive control strategy based on online grid impedance measurement is able to improve the system stability and adapt to variable feeder impedance. The passivity-based control of the grid inverters is another promising way to evaluate the system stability and offer general guidelines to properly shape the inverter impedance [14], [15]. 


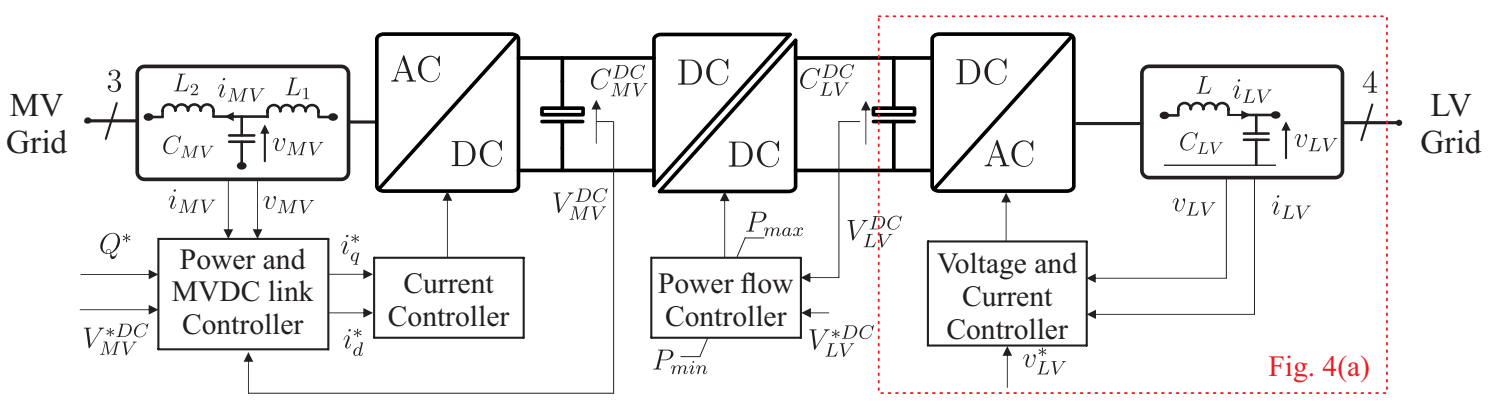

Fig. 3. Schematic diagram of a three-stage smart transformer.

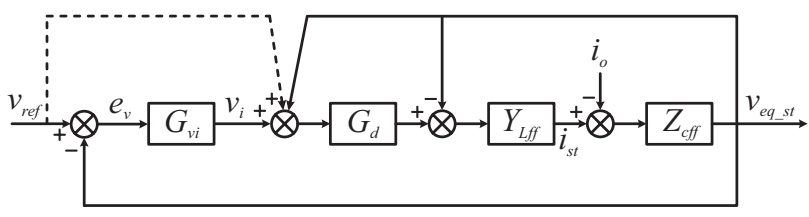

(a)

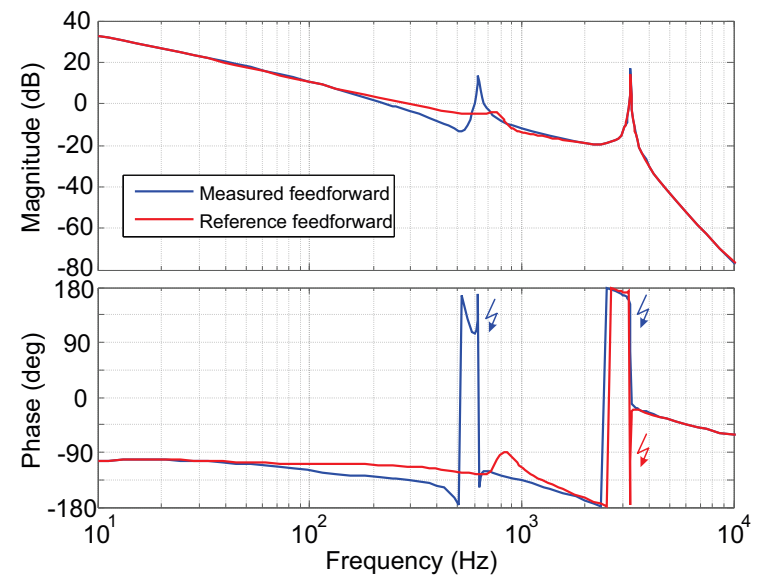

(b)

Fig. 4. Voltage control of the LV side DC/AC converter with different voltage feedforward: (a) schematic diagram, (b) open-loop Bode diagram for a DER inverter which is connected to the ST.

\section{B. Stability Analysis of a ST-fed Grid}

The ST is a three-stage solid-state transformer that adapts voltage between MV and LV grids while providing ancillary services to both utility and customers [10], for instance, voltage and current profiles improvement, balancing services in MV and LV grids, and stability improvement. The basic control structure of a three-stage ST is shown in Fig. 3. On the MV side, the ST controls the current from the MV grid to satisfy the active power request from the load and the reactive power request for the ancillary service. The DC/DC stage deals with the balancing between the two stages, namely the power flow between the MV grid and the LV grid. Due to the presence of DC-links, the power flow between MV and $\mathrm{LV}$ grids is decoupled, allowing the independent control of both side grids. On the LV side, the ST regulates the voltage waveforms on the LV grid while additional services can be offered by means of voltage control. Since the main purpose of this paper is to study the stability issue of LV grid, the other two stages and the rest of the grids are not taken into account thanks to the decoupling characteristic. To simplify the analysis and control design, the LV DC-link voltage is assumed to be constant.

The main objective of the LV side DC/AC converter is to control the voltage waveforms independently from the systems connected to the distribution grid and other disturbances. The detailed control diagram of the LV side DC/AC converter is depicted in Fig. 4a, in which an $L C$ filter is utilized as the AC side output filter, $Y_{L f f}$ and $Z_{c f f}$ are the admittance of the $L C$ inductor and the impedance of the $L C$ capacitor, respectively, $G_{v i}$ and $G_{d}$ are the transfer functions of the voltage controller and the computation \& PWM delay. Two voltage feedforward schemes are commonly used in voltagecontrolled applications to offer better performance [16], [17], which are the measured voltage feedforward (as the solid line) and the reference voltage feedforward (as the dash line). Depending on different voltage feedforward schemes, the equivalent output impedances of the ST are written by

$Z_{e q_{-} s t_{-} m f}(s)=\frac{Z_{c f f}(s)}{Z_{c f f}(s) Y_{L f f}(s)\left(1+G_{d}(s) G_{v i}(s)-G_{d}\right)+1}$.

$$
Z_{e q_{-} s t_{-} r f}(s)=\frac{Z_{c f f}(s)}{Z_{c f f}(s) Y_{L f f}(s)\left(1+G_{d}(s) G_{v i}(s)\right)+1} .
$$

where $Z_{\text {eq_st_mf }}$ and $Z_{\text {eq_st_rf }}$ are the equivalent impedances of ST with measured voltage feedforward and reference voltage feedforward, respectively. Ignoring line impedance, the equivalent grid impedance $Z_{e q_{-} g}$ of the ST-fed grid equals to $Z_{\text {eq_st_mf }}$ or $Z_{\text {eq_st_rf }}$, depending on the voltage feedforward schemes. Considering the local DER inverter uses the same control strategy of Fig. 1a, the schematic diagram with the consideration of $Z_{e q \_g}$ shown in Fig. 1b is still valid. By substituting the equivalent impedances of (2) and (3) into (1), the open-loop transfer functions of DER inverter considering the ST impedance can be obtained. Likewise the traditional grid, the stability of DER inverters is dependent on the equivalent ST/grid impedance. In order to reveal the potential instability in a ST-fed grid, two unstable cases are studied taking into account two different voltage feedforward schemes. The openloop Bode diagrams of the DER inverter with $Z_{e q_{-} s t} m f$ and $Z_{\text {eq_st_rf }}$ are shown in Fig. $4 \mathrm{~b}$, in which the stability issues are different. For the ST LV converter with reference voltage feedforward (red curve), the connected DER inverters are more likely to suffer from the resonance issue because the critical point only appears in the high frequency range. On the other 
hand, when the ST LV converter utilizes measured voltage feedforward (blue curve), the influence of the ST output filter is introduced and the connected DER inverters are subject to both resonance issue and harmonic instability [18], represented by oscillation and low-order harmonic distortion.

\section{Active Damping-Based Stabilization Methods}

One distinguished feature of ST is to reshape its output impedance and therefore accordingly modify the equivalent impedance of the ST-fed grid. As soon as the stability issues are detected, the ST impedance will be reshaped by means of voltage control, while the grid impedance $Z_{e q \_g}$ are being changed as well. According to (1), the changing of $Z_{e q_{-} g}$ leads to the alteration of phase margin of the DER inverter, which in fact offers a possibility to stabilize the DER inverters connected to the grid.

Two types of active damping methods are used aiming at reshaping the ST output impedance properties and stabilizing the grid-connected inverter of DER, which are: 1) for the ST LV converter with reference voltage feedforward, the multiloop-based active damping which is able to reshape the impedance within the Nyquist frequency [19]; 2) for the ST LV converter with measured voltage feedforward, the filterbased active damping which can adapt the impedance in certain frequency band [20]. From the analysis of Section II$\mathrm{B}$, it is known that the stability issues are subject to loworder harmonics and resonance. To identify the issues, a Discrete Fourier Transform (DFT) can be adopted and easily implemented into the voltage control shown in Fig. 5. In case the harmonic contents of the load current exceed the threshold $i_{h}$, the proposed active damping methods will be switched on. The detailed schematics of active damping methods are given and discussed in the following section.

\section{A. Multiloop-based Active Damping}

When the reference voltage feedforward is applied to the ST LV converter, the critical point usually situates at the resonant frequency, which could incur resonances of the LV grid. One of the intuitive solutions to address the stability issues related to the resonance is to use multiloop active damping method. For the voltage-controlled applications, an inductor current inner-loop is usually employed and the schematic diagram is shown in Fig. 5a, in which $k_{c}$ is the inner-loop gain. When the resonance is detected by the DFT, i.e. the harmonic contents of $i_{o}$ exceed the threshold at the resonant frequency, the inductor current inner-loop will be activated. As the gain of the inner-loop essentially introduces a virtual resistor to

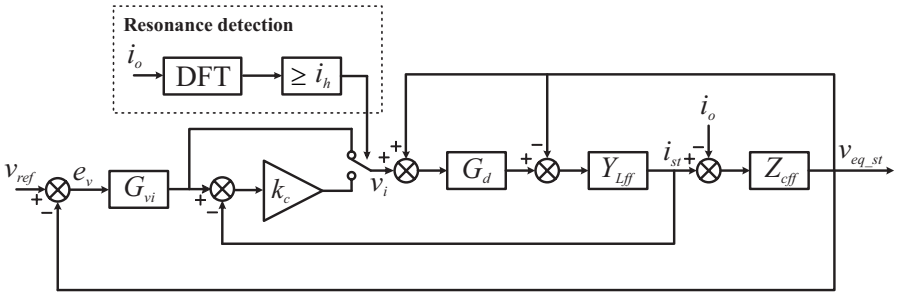

(a)

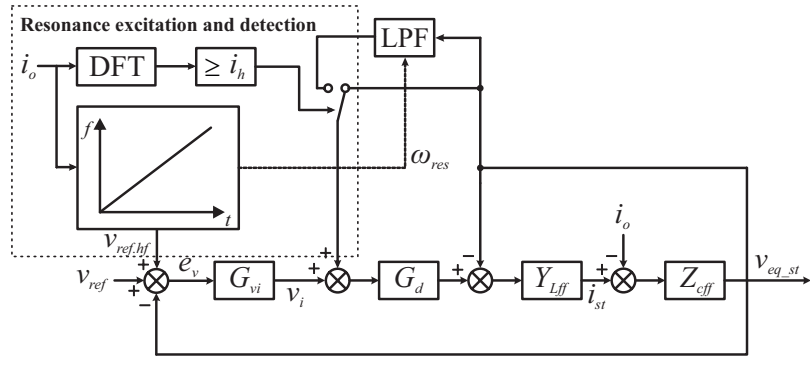

(b)

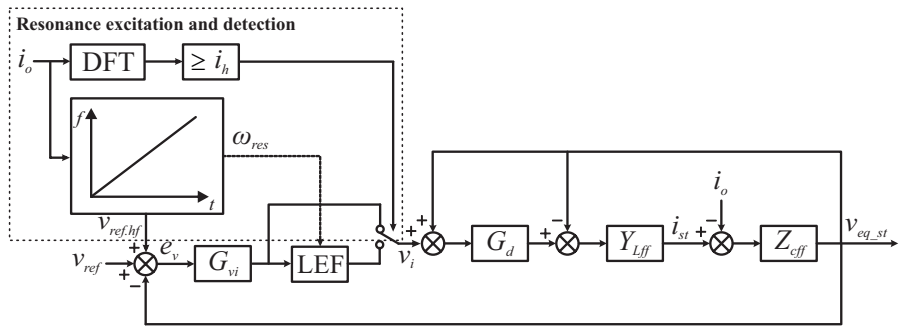

(c)

Fig. 5. Schematic Diagram of the ST voltage control utilizes different active damping methods: (a) Multiloop-based active damping, (b) lowpass filter-based active damping, and (c) lead element filter-based active damping.

the system, the resonance peaks of both the ST converter and the DER inverter can be alleviated when a reasonable damping ratio is given. Based on Fig. 5a, the equivalent output impedance of the ST LV converter is given in (4). Substituting (4) into (1), the transfer function of the DER grid inverter considering the effect of the grid impedance can be obtained, and the Bode diagram is shown in Fig. 6a. To better evaluate the performance improvement, the dynamic response of the DER inverter connected to a ST-fed grid without the active damping method is shown by the red curve as well. It is worth to mention that both the control system of ST LV side converter and the one of DER inverter are pre-designed with sufficient stability margins. From Fig. 6a, if the active damping method is not used, it is seen that there is a positive $-180^{\circ}$ crossing in the neighborhood of the resonant frequency when

$$
\begin{gathered}
Z_{e q_{-} s t_{-} I L P}(s)=\frac{Z_{c f f}(s)\left(1+k_{c} Y_{L f f}(s) G_{d}(s)\right)}{Z_{c f f}(s) Y_{L f f}(s)\left(1+k_{c} G_{d}(s) G_{v i}(s)\right)+k_{c} Y_{L f f}(s) G_{d}(s)+1} . \\
Z_{e q_{-} s t_{-} L P F}(s)=\frac{Z_{c f f}(s)}{Z_{c f f}(s) Y_{L f f}(s)\left[1+G_{d}(s)\left(G_{v i}(s)-G_{L P F}(s)\right)\right]+1} . \\
Z_{\text {eq_st_LEF }}(s)=\frac{Z_{c f f}(s)}{Z_{c f f}(s) Y_{L f f}(s)\left(1+G_{d}(s) G_{v i}(s) G_{L E F}(s)-G_{d}\right)+1} .
\end{gathered}
$$




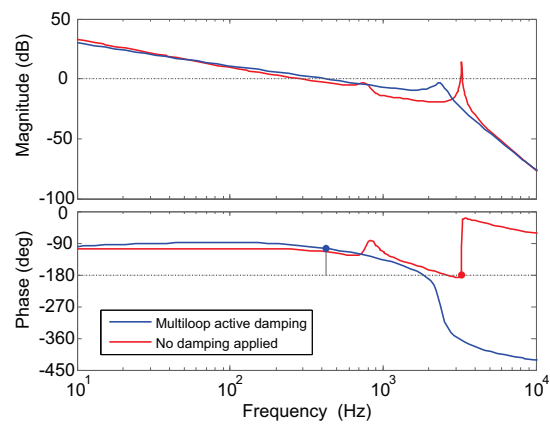

(a)

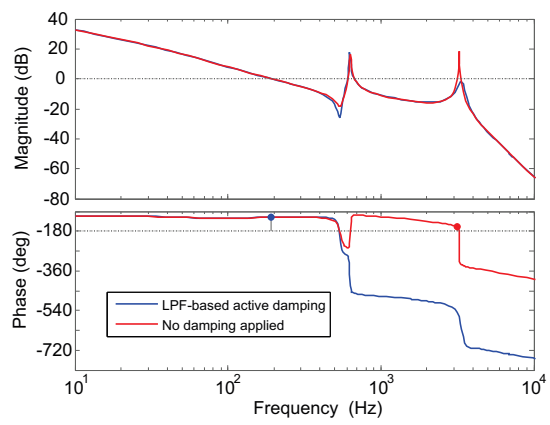

(b)

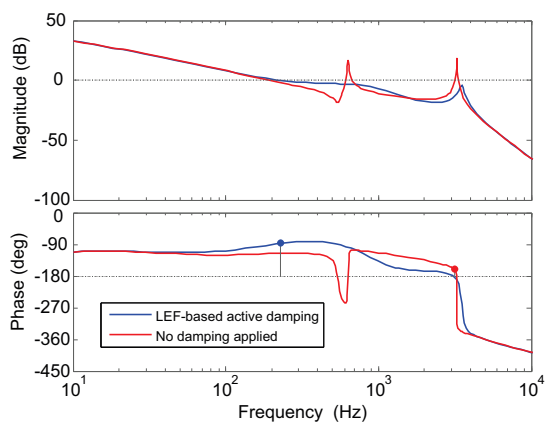

(c)

Fig. 6. Bode diagrams of the grid-connected inverter of DER considering the effects of the grid impedance (ST output impedance): (a) a inner-loopbased active damping is applied to the ST, (b) a LPF-based active damping is applied to the ST, and (c) a LEF-based active damping is applied to the ST.

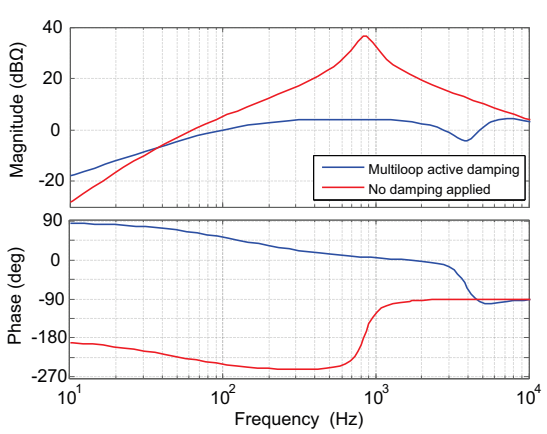

(a)

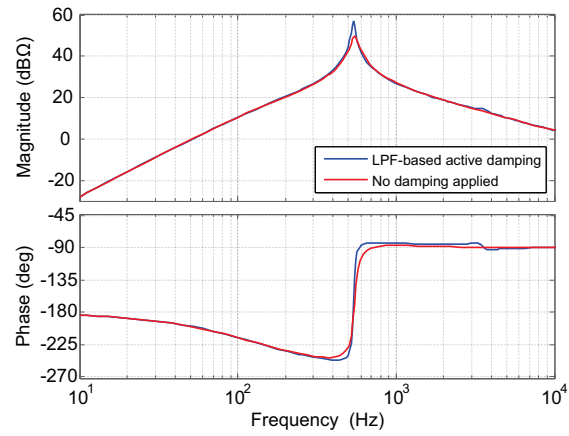

(b)

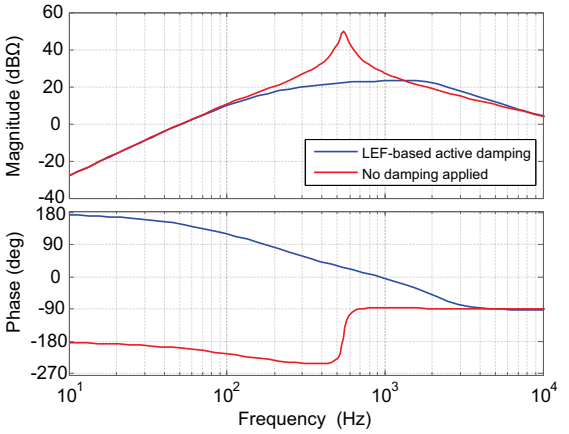

(c)

Fig. 7. Properties of the ST output impedance when: (a) a inner-loop-based active damping is applied to the ST, (b) a LPF-based active damping is applied to the ST, and (c) a LEF-based active damping is applied to the ST.

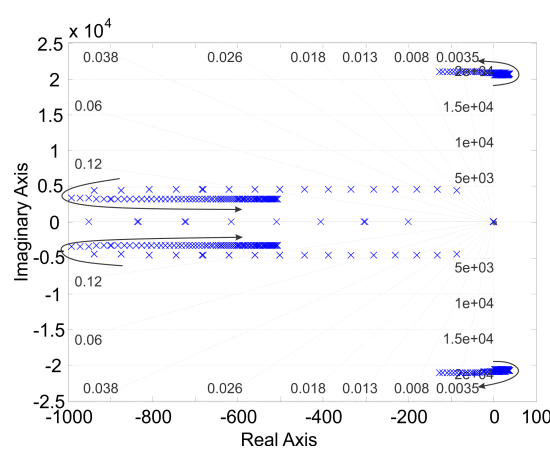

(a)

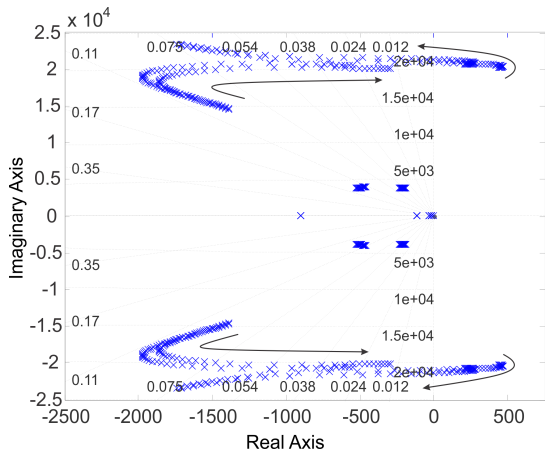

(b)

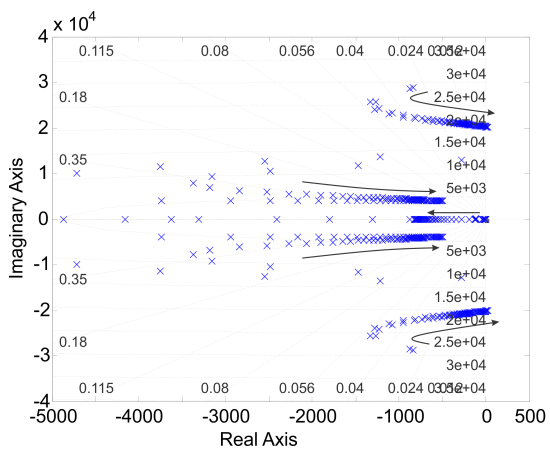

(c)

Fig. 8. Root locus of the DER inverter when different active damping methods are applied to the ST: (a) Multiloop-based active damping increasing $k_{c}$, (b) low-pass filter-based active damping increasing $\omega_{c}$, and (c) lead element filter-based active damping increasing $\omega_{f}$.

the magnitude above $0 \mathrm{~dB}$ (red curve). Hence, it indicates that the system is unstable according to the Nyquist stability criterion. After the inner-loop-based active damping is plugged in, it is seen that the resonance peak of the DER inverter has been damped and therefore the $-180^{\circ}$ is crossed only once while the magnitude at that point is negative. Therefore, a stable operation is achieved in this case (minimum phase margin is $64.4^{\circ}$ ).

The impedance properties of the ST LV side converter are shown in Fig. 7a. The red curve represents the equivalent impedance of the ST without any active damping method, while the blue curve represents the impedance with the innerloop-based active damping. It is shown that the impedance magnitude at the resonant frequency of the ST converter is reduced and the others at the low-order harmonic range are suppressed as well, while the impedance magnitude in the low-frequency range has been amplified. From the point of view of ST voltage control, a smaller impedance at the resonant frequency is beneficial to alleviate the resonance issues. However, the impedance increases in the low-frequency range could deteriorate the control accuracy. A tradeoff between the 
damping effect and the voltage control performance is hereby necessary.

\section{B. Filter-based Active Damping}

When the measured voltage feedforward is applied to the ST LV converter, the critical points lie not only at the resonant frequency but also in the low-order harmonic range, which leads to harmonic instability. A practical technique to address the resonance/harmonic-related stability issues is to use the filterbased active damping methods. Compared to the multiloopbased active damping, the filter-based methods preserve the closed-loop bandwidth so that the control performance can be maintained [20]. With these considerations, Low-Pass Filter (LPF) and Lead Element Filter (LEF)-based active damping methods are conisdered in the following scenarios. Both methods can damp the resonance peak and modify the magnitude and phase of certain frequency band so that they are able to mitigate the both critical points shown in Fig. 4. In addition, considering robustness, LPF and LEF are not sensitive to the variation of resonant frequency. To well tune the filter parameters, an estimator of the resonant frequency of the LV grid by means of frequency sweeping (e.g. [21], [22]) is needed for ST. As shown in Fig. 5b and 5c, the ST LV side converter is able to excite the resonance of $\mathrm{LV}$ grid by generating harmonic voltage perturbations within all frequency range. The detected resonant frequency is adopted by the LPF and the LEF for the parameters tuning. The optimal ranges of two filters' parameters regarding the resonant frequency are given in Section IV.

A LPF attenuates the magnitude and introduces a phase delay in a frequency band above its cutoff frequency. These two features can be beneficial to shape the ST equivalent impedance in the band around the resonant frequencies of ST converter and DER inverter. Fig. 5b shows the schematic diagram of the ST voltage control with the LPF-based active damping. Once the harmonic stability or the resonance is detected by the DFT, namely, the harmonic contents of $i_{o}$ exceed the threshold at the interested frequencies, the measured voltage feedforward with a LPF will be implemented. Based on the schematic diagram, the ST equivalent impedance is given in (5) and the transfer function as well as the Bode diagram (show in Fig. 6b) of the DER grid inverter considering the grid impedance can be obtained by combining (1) and (5). Here, a second-order LPF is adopted, the transfer function of which is given by

$$
G_{L P F}(s)=\frac{\omega_{c}^{2}}{s^{2}+2 D \omega_{c} s+\omega_{c}^{2}} .
$$

where $\omega_{c}$ is the cutoff frequency and $D=1 / \sqrt{2}$ is used in the study. Assuming the resonant frequency of the LV grid $\omega_{\text {res }}$ is known, $\omega_{c}=0.75 \omega_{\text {res }}$ is chosen in this case. It is observed in Fig. 6b that the LPF-based active damping is able to introduces a phase delay in the low-frequency range and attenuate the resonance peak of DER inverter (the peak at $3350 \mathrm{~Hz}$ ). As a result, the $-180^{\circ}$ crossing in the low-frequency range is shifted outside the frequency band with a magnitude above $0 \mathrm{~dB}$ and the resonance peak of DER inverter is damped with a negative magnitude (blue curve). A stable operation is achieved in this case (minimum phase margin is $63.5^{\circ}$ ). The impedance properties of the ST LV side converter are shown in Fig. $7 b$, in which the original properties are well maintained within the entire Nyquist frequency. Thus, the LPF-based active damping method can hold the characteristics of the original ST voltage control system, and this is especially beneficial for the fundamental voltage control and low-order harmonic elimination.

A LEF introduces a phase lead in a certain frequency band. By using the LEF-based active damping, the impedance properties of ST in the phase-lead-compensation frequency band can be reshaped. The schematic diagram of the ST voltage control with the LEF-based active damping is depicted in Fig. 5c. As soon as the harmonic stability or the resonance is detected, a LEF will be plugged into the voltage control forward channel. The equivalent ST output impedance is given in (6) based on Fig. 5c. The transfer function of the DER inverter considering the effect of the grid impedance can be obtained by substituting (6) into (1), and its Bode diagram is shown in Fig. 6c. Here, the transfer function of LEF is given by

$$
G_{L E F}(s)=\frac{1 / \omega_{f} s+1}{\alpha / \omega_{f} s+1} .
$$

where $\omega_{f}$ and $\alpha$ determine the phase-lead-compensation frequency band and the phase-lead-compensation angle, respectively. A relation $\alpha=\tan \left(\frac{\pi}{4}-\frac{\phi_{\max }}{4}\right)$ holds, $\phi_{\max }=30^{\circ}$. $\omega_{f}=0.55 \omega_{\text {res }}$ is chosen in this case. It can be seen in Fig. $6 c$ that the LEF-based active damping changes the phases of the phase-lead-compensation band and damps the resonance peaks. Due to the phase change, it causes the phase response crossing $-180^{\circ}$ away from the critical frequency range and stabilizes the overall system (minimum phase margin is $87.4^{\circ}$ ). The impedance properties shown in Fig. 7c shows that the LEF-based active damping can damp the resonance of the ST converter and maintain the control characteristics except the phase-lead-compensation band.

\section{Design of Active Damping Parameters}

The active damping proposed in literature is usually to suppress the resonance and improve the stability margin of inverter-interfaced DER. Design criteria have been given to achieve these targets [20], [23]. Nevertheless, the active damping methods being implemented in the ST LV inverter aims at a different target. Instead of stabilizing one DER itself, the main purpose is to stabilize all the DER inverters in the LV grid by means of ST. As a result, the stability of the DER grid inverter is the basic criterion. In addition, there are other demands being taken into consideration, for example the bandwidth and control accuracy of the ST. In general, the influence of the active damping on the ST control is another main concern because a stiff ST voltage is the key factor of the ST-fed grid operation. Therefore, the parameters of the active damping (e.g. $k_{c}, \omega_{c}$, and $\omega_{f}$ ) are tuned considering two criteria: 1) the parameters are effective enough to stabilize the DER inverters; 2) the original control properties of the ST voltage control in terms of dynamics and steady-state control 


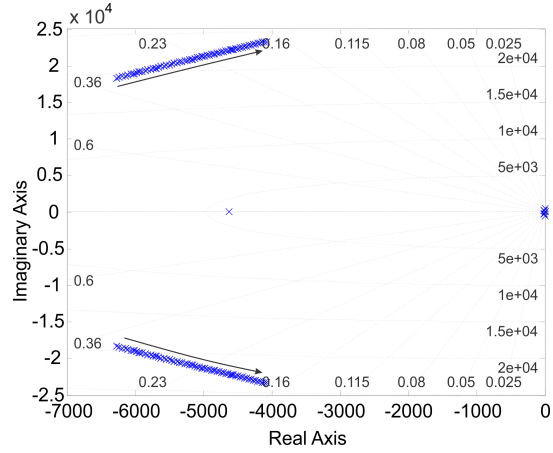

(a)

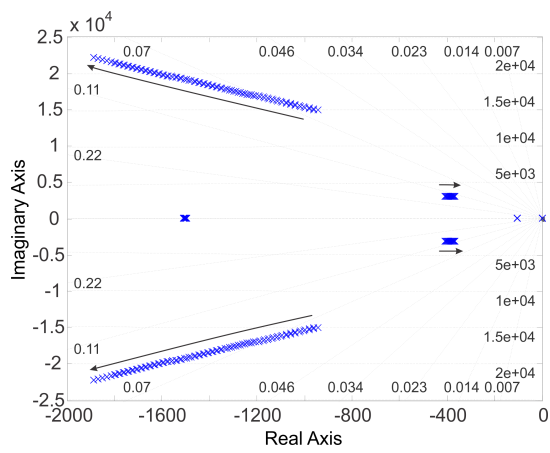

(b)

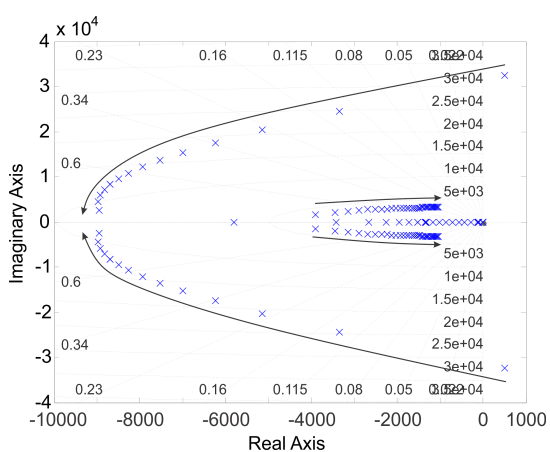

(c)

Fig. 9. Root locus of the ST LV side converter when different active damping methods are applied: (a) Multiloop-based active damping increasing $k_{c}$, (b) low-pass filter-based active damping increasing $\omega_{c}$, and (c) lead element filter-based active damping increasing $\omega_{f}$.

accuracy should fulfill the grid requirements and should not be affected by the active damping methods. To avoid the impacts of other instability factors, a pre-design of the ST LV side voltage controller and the DER current controller was carried out, ensuring phase margins between $45^{\circ}$ and $60^{\circ}$.

\section{A. Multiloop-based Active Damping}

The choice of the inner-loop gain is a typical tradeoff between the stability requirement of DER inverter and the control accuracy of ST LV side converter. As being pointed out in Section III and seen in Fig. 7a, the introduce of the current inner-loop dramatically reshapes the equivalent ST output impedance. With the increasing of $k_{c}$, the equivalent impedance of the low-frequency range increases accordingly, which will amplify the disturbances and harmonics [19]. The pole-zero map of the DER inverter including the effects of grid impedance is shown in Fig. 8a, where $k_{c}$ increases from 1 to 75 . It can be seen that a pair of unstable poles are moved from the right-half plane to the left, proofing the improvement of the stability. In addition, the pole-zero map of the ST converter using the inner-loop-based active damping is shown in Fig. 9a. It is seen that the control bandwidth increases while the damping ratio decreases by increasing $k_{c}$. To sum up, a higher $k_{c}$ results in a more stable DER grid inverter but compromises the control performance in terms of lowfrequency disturbances and harmonics rejection. In a practical ST-fed grid, the value of $k_{c}$ should therefore be limited in order to guarantee good voltage waveform that can meet the requirements of the grid codes.

\section{B. Filter-based Active Damping}

Different from the multiloop-based active damping, the LPF-based active damping changes the equivalent ST output impedance in a limited way. Especially in the lowfrequency range, both the magnitude and phase response of the impedance remain the same as the one without damping. A pole-zero map of the DER inverter considering the grid impedance effects is presented in Fig. 8b, in which the cutoff frequency $\omega_{c}$ changes from $0.65 \omega_{\text {res }}$ to $0.95 \omega_{\text {res }}$. It is seen that two pairs of unstable poles move from the right-half plane to the left, while another two pairs of stable poles are shifted towards the imaginary axis, when $\omega_{c}$ increases. From the polezero map of the ST converter using LPF-based active damping, shown in Fig. 9b, it can be seen that the control bandwidth of the ST increases when $\omega_{c}$ increases, indicating a higher $\omega_{c}$ offers the ST better voltage dynamics. In summary, the stability requirement of the DER inverter is the main index for the tuning of $\omega_{c}$. All the dominant poles of the DER inverters must be in the left-half plane away from the imaginary axis. From the pole-zero maps, $\omega_{c}$ of $\left[0.75 \omega_{\text {res }}, 0.9 \omega_{\text {res }}\right]$ is preferred to be the optimal range. The optimal range of the cutoff frequency can be used in conjunction with the resonant frequency obtained from the resonance estimator (illustrated in Fig. 5b), enabling the adaptive feature to the variation of LV grid.

The LEF-based active damping changes the magnitude and phase of the equivalent ST output impedance within the phaselead-compensation frequency band. To mitigate the impact on the low-frequency range (usually up to $750 \mathrm{~Hz}$ ), the phaselead-compensation frequency $\omega_{f}$ should be higher than this range. Nevertheless, a higher $\omega_{f}$ could lead to instability of the DER inverter, seen from the pole-zero map shown in Fig. 8c. In this map, two pairs of poles move towards the imaginary axis till the right-half plane, when $\omega_{f}$ increases from $0.25 \omega_{\text {res }}$ to $0.5 \omega_{\text {res }}$. Meanwhile, from the pole-zero map of the ST converter using LEF-based active damping which shown in Fig. 9c, the control bandwidth, damping factor, and stability margin of the ST voltage control system reduce significantly when $\omega_{f}$ is increasing. With these considerations, provided $\omega_{f}$ is higher than the frequency of interested in the lowfrequency range, the lowest $\omega_{f}$ would be expected to provide good ST control performance and stabilization effect. From the pole-zero maps and the requirements of the application, $\omega_{c}$ of $\left[0.35 \omega_{r e s}, 0.45 \omega_{r e s}\right]$ is chosen to be the optimal range, and can be used together with the resonant frequency obtained from the resonance estimator (illustrated in Fig. 5c).

In general, both LPF- and LEF-based active damping methods can achieve comparable performance with the optimal parameters. The design of the LPF is much easier than that of the LEF and meanwhile the influences of LPF-based active damping on the ST performance is minor. On the other hand, the LEF-based active damping shows better damping effects 


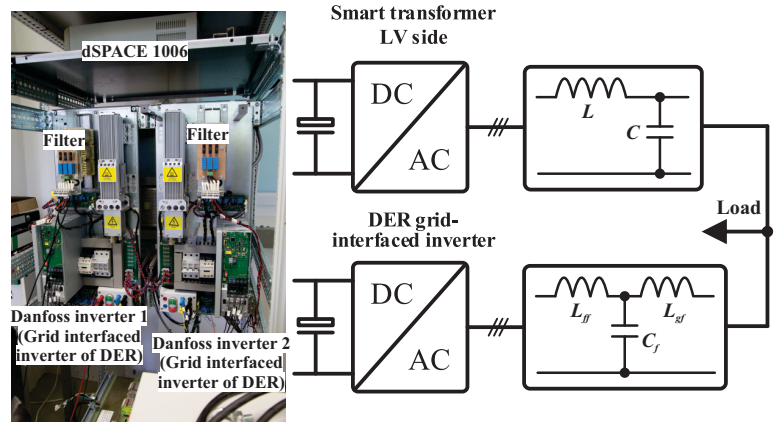

Fig. 10. Laboratory setup and its system configuration.

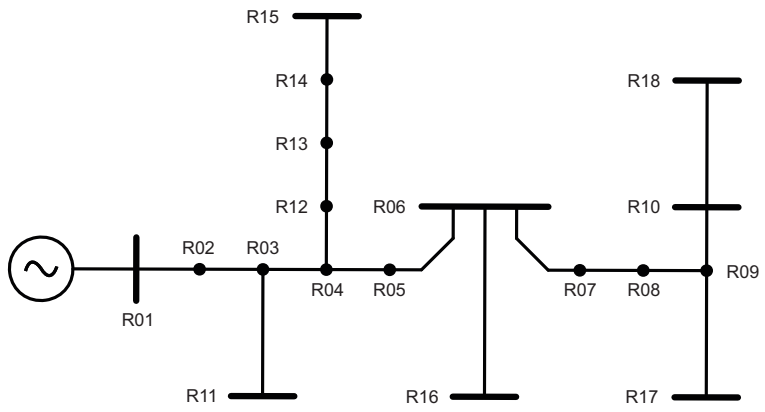

Fig. 11. CIGRE benchmark microgrid, adapted from [24].

on resonances. To summarize, the LPF-based active damping method is recommended in the harmonic instability condition, while in some severe cases like both resonance and harmonic distortion occur, the LEF-based active damping method can be an efficient alternative.

\section{VERIFICATION RESULTS}

To better study the stability issues of a ST-fed grid, a simplified ST-fed power system consisting of two commercial inverters and linear load has been developed in the laboratory (shown in Fig. 10). The validity of the proposed active damping methods has been experimentally verified by the setup. Furthermore, a CIGRÉ 18-Bus benchmark microgrid (shown in Fig. 11) has been implemented in a RTDS simulator, with the ST being the voltage supply and two DERs as well as local loads being connected to the microgrid, to show the effectiveness of the proposed methods in an actual microgrid. The verification results are presented in the following sections.

\section{A. Simulation Results}

The resonance estimation method of Fig. 5 is tested by MATLAB/Simulink with the aid of the PLECS tool box. The system configuration of Fig. 10 is used, and the system parameters are listed in Table I. The frequency sweeping is implemented in the voltage control of ST to detect the resonant frequency of LV grid, with an magnitude of 0.5 p.u. harmonic injection and the frequency variation of $23550 \mathrm{rad} / \mathrm{s}$. The sweeping frequency and the corresponding $\mathrm{LV}$ side current are shown in Fig. 12. It can be seen that the resonance of the LV grid is excited by the method at $3450 \mathrm{rad}$, which can be used in the parameter tuning of the filter-based active damping.

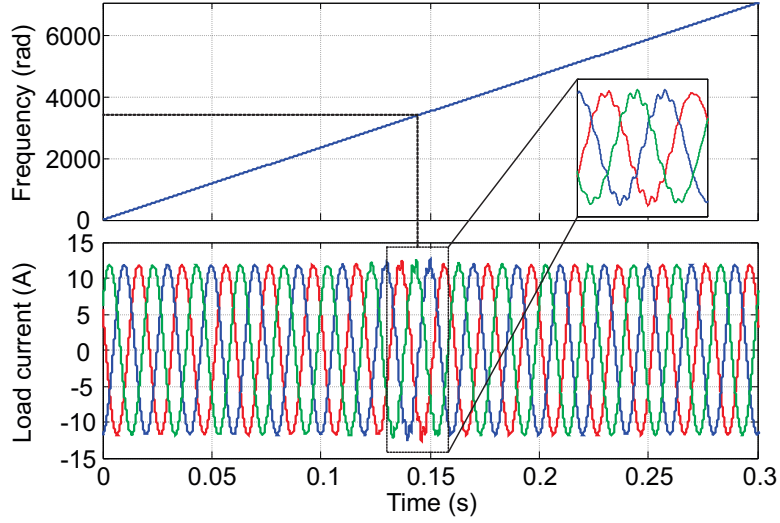

Fig. 12. Sweep to excite the resonance of LV grid: (a) Sweeping frequency and (b) current of LV side of ST.

\section{B. Experimental Verification}

To validate the effectiveness of the proposed methods, a ST-fed grid is tested in the experimental setup. The system configuration of Fig. 10 is taken, the current control scheme of Fig. $1 \mathrm{~b}$ and the voltage control schemes of Fig. 4a are implemented in the DER grid inverter and the ST LV side converter, respectively. Two Danfoss FC302 inverters are used: one for the ST LV side converter, and the other one for the grid-interfaced inverter of DER. A dSPACE 1006 is used to achieve the voltage/current control strategies as well as the proposed stabilization solutions in real-time. The system parameters are listed in Table I. Two standard PI controllers are employed as the current controller $G_{c i}$ and the voltage controller $G_{v i}$. A pre-design of both $G_{c i}$ and $G_{v i}$ is carried out to make sure the stability margins of the DER inverter (standalone) and the ST LV side converter (without DER inverter being connected) are between $45^{\circ}$ and $60^{\circ}$.

Two case studies are carried out to test the effectiveness of the active damping-based stabilization solutions. Firstly, when the reference voltage feedforward is applied to the ST, an unstable case in terms of resonance occurs. From Fig. 13a, it is seen that both the ST LV side voltage and the DER output current are oscillated initially. To mitigate this issue, the inner-loop-based active damping of Fig. 5a is utilized at $0 \mathrm{~s}$ (the switch signal of active damping is plotted by the red curves in all the cases). Both the oscillations of the ST voltage and the DER current are well damped as soon as the damping is enabled. For better comparison, the zoomed figure of the resonating condition and the stable condition are shown at the left side and the right side, respectively. Secondly, when the measured voltage feedforward is applied to the ST, an unstable case in terms of harmonic distortion happens. From Fig. 13b, it is shown that both the ST LV side voltage and the DER output current are unstable and highly distorted at the very beginning. At $0 \mathrm{~s}$, the LPF-based active damping method of Fig. $5 b$ is activated in the ST. It can be seen that both the ST voltage and the DER current are effectively stabilized and both waveforms recover their nominal states with good power quality in very short term. The stabilization effect can also be achieved by the LEF-based active damping method of Fig. 5c. In Fig. 13c, the LEF-based active damping method is activated 


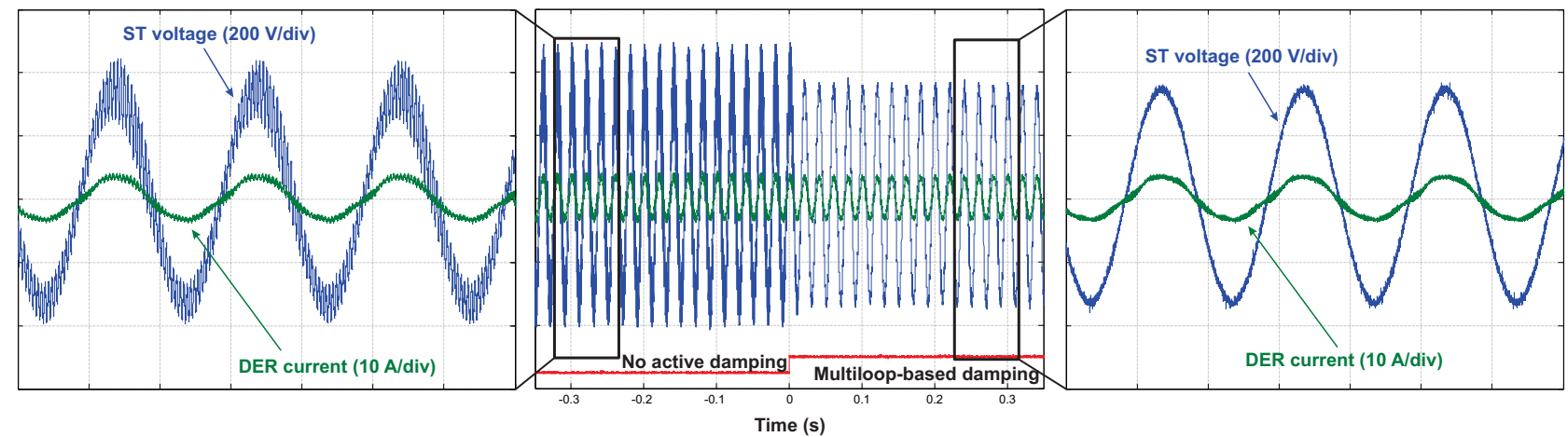

(a)

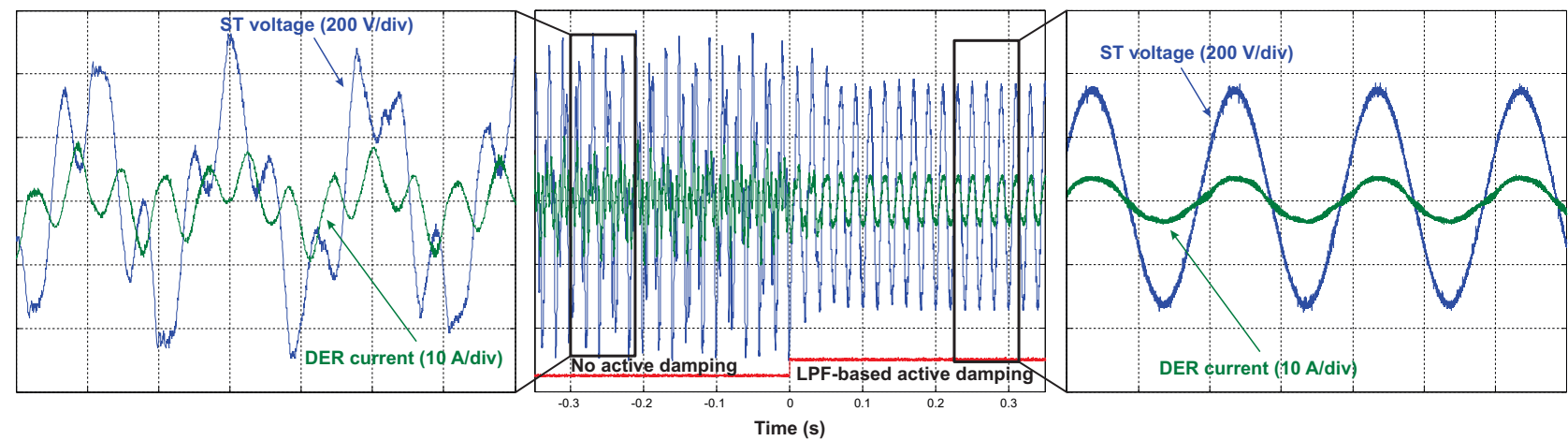

(b)
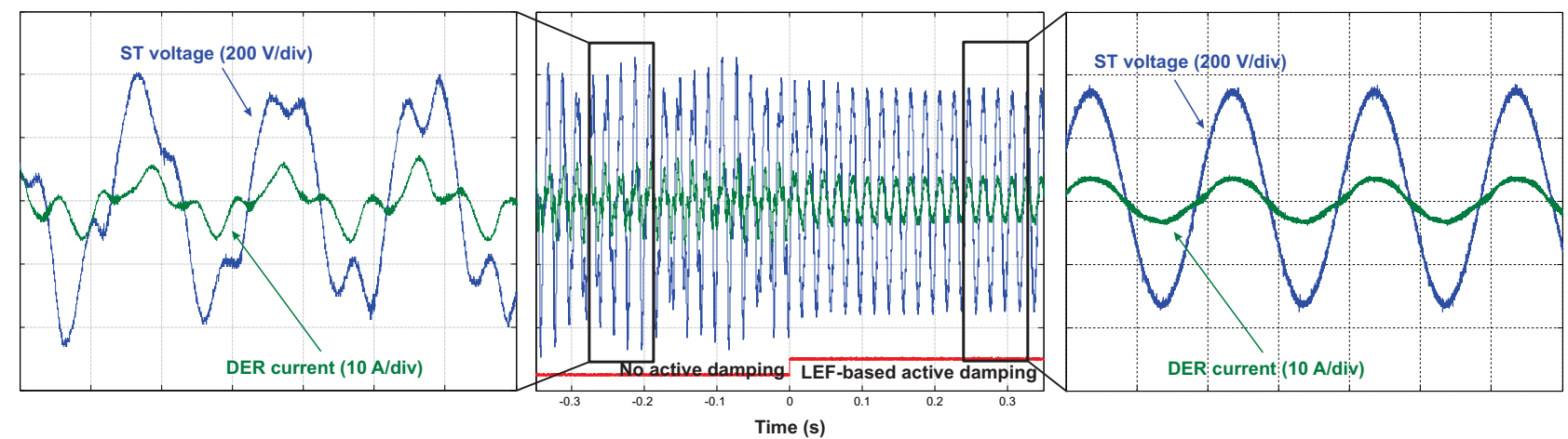

(c)

Fig. 13. Experimental waveforms of ST LV side voltage and DER current with/without different active damping-based stabilization methods under the resonant/harmonic instability conditions: (a) multiloop-based active damping method, (b) LPF-based active damping method, and (c) LEF-based active damping method.

in the ST at $0 \mathrm{~s}$, both the ST LV side voltage and the DER current are immediately stabilized and quickly recover to their nominal states. The zoomed figures of the harmonic instability condition and the stable condition are shown at the left side and the right side, respectively.

\section{Benchmark Grid Evaluation in RTDS}

A CIGRÉ 18-Bus benchmark grid (shown in Fig. 11) is employed and simulated in RSCAD, the RTDS software. The cable configuration including cable types and lengths of the benchmark grid are presented in TABLE II and TABLE III. The LV side of ST is connected to Bus R01, being the voltage source of the benchmark microgrid. Two grid inverter interfaced DERs are connected to the Buses of R16 and R18, and their nominal power ratings are $25.68 \mathrm{kVA}$ and $17.98 \mathrm{kVA}$, respectively. To meet the modern grid codes, the reactive power injection of DER is considered, and the Power Factor (PF) of both grid-interfaced inverters is 0.95 in this study. The control strategies mentioned in Section II have been used in the case study. Note that the stability margins of the ST (without DER being connected) and the DERs (standalone) are designed to be between $45^{\circ}$ and $60^{\circ}$. Several constant power loads are connected to the Buses of R11, R15, and R16, and their power ratings are $50 \mathrm{kVA}, 5 \mathrm{kVA}$, and $5 \mathrm{kVA}$, with $\mathrm{PF}$ equals to 0.99 .

Firstly, the reference voltage feedforward is applied to the ST, when DERs are connected to the grid, leading to an unstable case in terms of resonance (shown in Fig. 14). At $0.175 \mathrm{~s}$, the multiloop-based active damping of Fig. 5a is activated. It can be seen that the voltage oscillation of Bus 


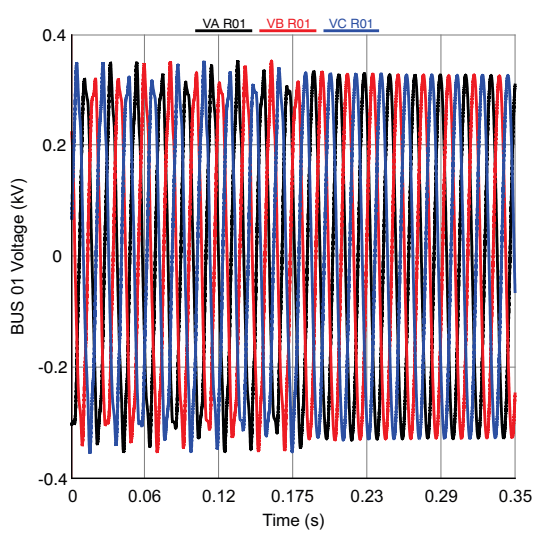

(a)

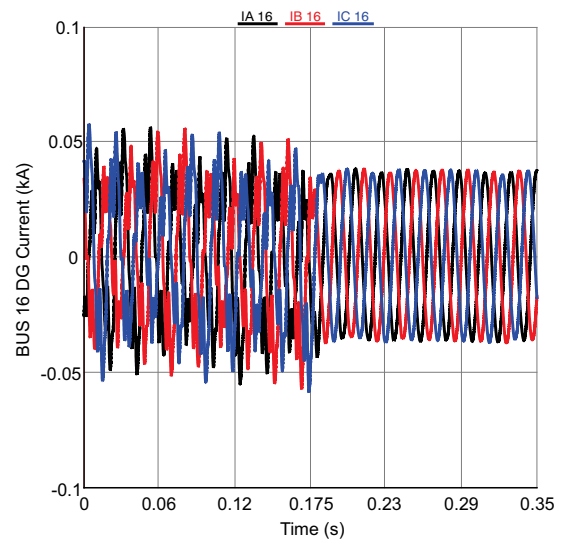

(b)

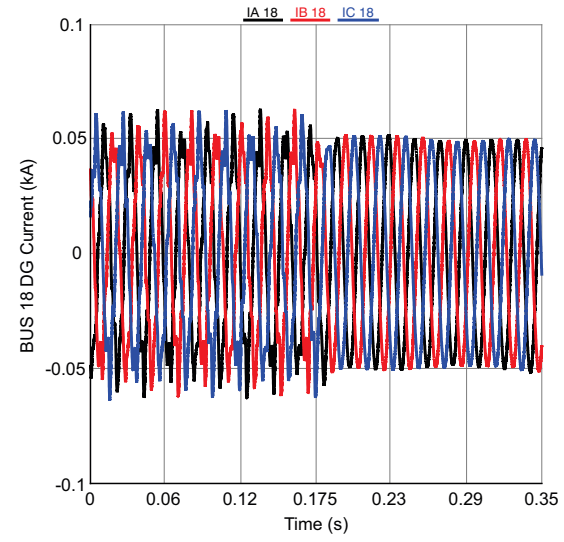

(c)

Fig. 14. RTDS results of a ST-fed grid with/without inner-loop-based active damping under resonant condition: (a) Bus R01 voltage, (b) current injection at Bus R16, and (c) current injection at Bus R18.

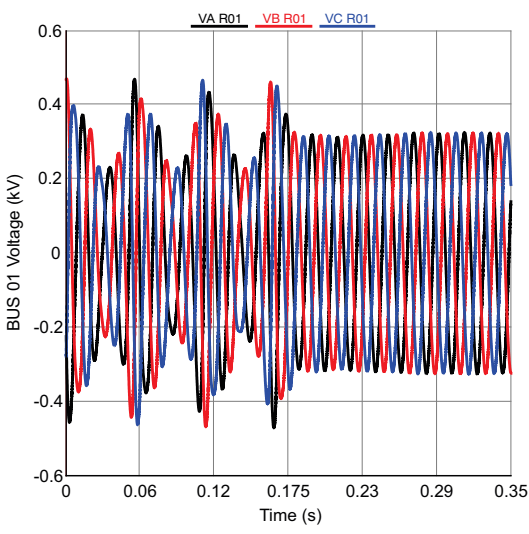

(a)

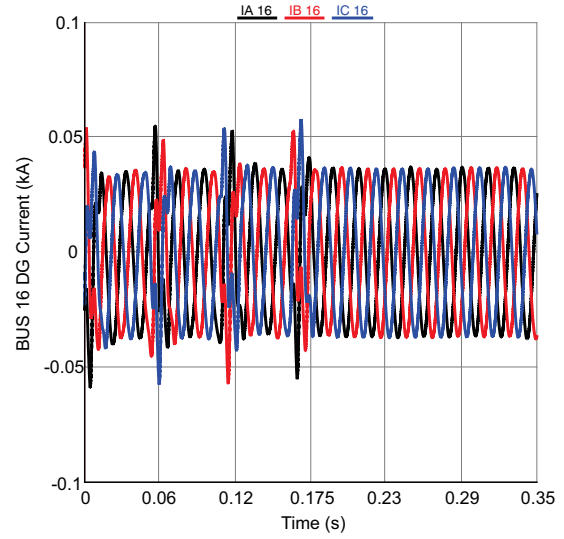

(b)

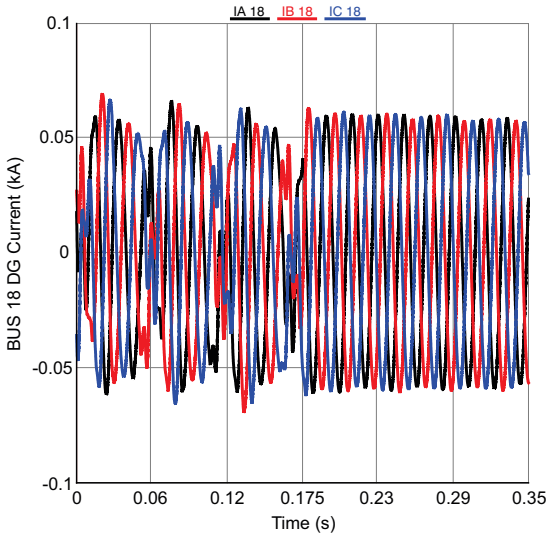

(c)

Fig. 15. RTDS results of a ST-fed grid with/without filter-based active damping under harmonic instability condition: (a) Bus R01 voltage, (b) current injection at Bus R16, and (c) current injection at Bus R18.

TABLE I

SYSTEM PARAMETERS

\begin{tabular}{lll}
\hline \hline Symbol & \multicolumn{1}{c}{ Quantity } & \multicolumn{1}{c}{ Value } \\
\hline$f_{c}$ & switching frequency & $10 \mathrm{kHz}$ \\
$V_{d c}$ & dc-link voltage & $650 \mathrm{~V}$ \\
$v_{n}$ & rated voltage of inverters & $3 \times 380 \mathrm{~V}(\mathrm{rms})$ \\
$S_{n}$ & rated power of inverters & $4 \mathrm{~kW}$ \\
$L$ & filter inductance of ST & $5.03 \mathrm{mH}$ \\
$C$ & filter capacitance of ST & $10 \mu \mathrm{F}$ \\
$L_{f f}$ & inverter-side filter inductance of DER & $5.03 \mathrm{mH}$ \\
$C_{f f}$ & filter capacitance of DER & $10 \mu \mathrm{F}$ \\
$L_{g f}$ & grid-side filter inductance of DER & $0.5 \mathrm{mH}$ \\
\hline \hline
\end{tabular}

R01 is damped immediately and the resonances as well as oscillations of the DER connected to the Buses of R16 and R18 are stabilized within a few cycles (less than $100 \mathrm{~ms}$ ).

Secondly, the measured voltage feedforward is employed by the ST. The instability is featured by low-order harmonic dis- tortion of voltage and current when two DERs are connected to the grid (shown in Fig. 15). At $0.175 \mathrm{~s}$, the filter-based active damping of Fig. 5b is implemented in the ST control, which turns out the harmonic distortions as well as oscillations of both voltage and current are phased out in about two cycles (less than $50 \mathrm{~ms}$ ). The results show that the proposed active damping methods can stabilize the benchmark grid under various conditions, in particular the ancillary services (like reactive power compensation) are offered by the local DERs.

\section{CONCLUSIONS}

In this paper, the stability issue of grid-connected inverter in a ST-fed grid is studied and three active damping methods are proposed and implemented in the ST to stabilize the grid inverter as well as the grid. The proposed active damping methods implemented in a ST are able to reshape the equivalent grid impedance. Implementing the active damping in a centralized way, this procedure can effectively improve the stability of the DER grid-connected inverters and meanwhile reduces the design effort of the local control systems. Comprehensive control design and analysis are presented in this 
TABLE II

LINE CONFIGURATION

\begin{tabular}{lcccl}
\hline \hline Name & From & To & Cable & Length (m) \\
\hline$L-01$ & $R-01$ & $R-02$ & $U G-01$ & 35 \\
$L-02$ & $R-02$ & $R-03$ & $U G-01$ & 35 \\
$L-03$ & $R-03$ & $R-04$ & $U G-01$ & 35 \\
$L-04$ & $R-04$ & $R-05$ & $U G-01$ & 35 \\
$L-05$ & $R-05$ & $R-06$ & $U G-01$ & 35 \\
$L-06$ & $R-06$ & $R-07$ & $U G-01$ & 35 \\
$L-07$ & $R-07$ & $R-08$ & $U G-01$ & 35 \\
$L-08$ & $R-08$ & $R-09$ & $U G-01$ & 35 \\
$L-09$ & $R-09$ & $R-10$ & $U G-01$ & 35 \\
$L-10$ & $R-03$ & $R-11$ & $U G-04$ & 30 \\
$L-11$ & $R-04$ & $R-12$ & $U G-02$ & 35 \\
$L-12$ & $R-12$ & $R-13$ & $U G-02$ & 35 \\
$L-13$ & $R-13$ & $R-14$ & $U G-02$ & 35 \\
$L-14$ & $R-14$ & $R-15$ & $U G-02$ & 30 \\
$L-15$ & $R-06$ & $R-16$ & $U G-06$ & 30 \\
$L-16$ & $R-09$ & $R-17$ & $U G-04$ & 30 \\
$L-17$ & $R-10$ & $R-18$ & $U G-05$ & 30 \\
\hline
\end{tabular}

TABLE III

CABLE TYPES

\begin{tabular}{clll}
\hline \hline Type & $R^{\prime}(\Omega / \mathrm{km})$ & $X^{\prime}(\Omega / \mathrm{km})$ & $R^{\prime} / X^{\prime}$ \\
\hline$U G-01$ & 0.163 & 0.136 & 1.199 \\
$U G-02$ & 0.266 & 0.151 & 1.762 \\
$U G-04$ & 1.541 & 0.206 & 7.481 \\
$U G-05$ & 1.111 & 0.195 & 5.697 \\
$U G-06$ & 0.569 & 0.174 & 3.270 \\
\hline \hline
\end{tabular}

paper, considering the control performance and stability of the DER and the ST. Case studies of the proposed active damping methods in a ST-fed microgrid are performed in both the experimental platform and the RTDS. The results prove the effectiveness of those methods in dealing with the resonance and instability issues.

\section{REFERENCES}

[1] N. Bottrell, M. Prodanovic, and T. C. Green, "Dynamic stability of a microgrid with an active load," IEEE Trans. Power Electron., vol. 28, no. 11, pp. 5107-5119, Nov. 2013.

[2] B. Wen, D. Boroyevich, R. Burgos, P. Mattavelli, and Z. Shen, "Smallsignal stability analysis of three-phase ac systems in the presence of constant power loads based on measured d-q frame impedances," IEEE Trans. Power Electron., vol. 30, no. 10, pp. 5952-5963, Oct. 2015.

[3] J. L. Agorreta, M. Borrega, J. Lopez, and L. Marroyo, "Modeling and control of $\mathrm{n}$-paralleled grid-connected inverters with lcl filter coupled due to grid impedance in pv plants," IEEE Trans. Power Electron., vol. 26, no. 3, pp. 770-785, Mar. 2011.

[4] A. A. Rockhill, M. Liserre, R. Teodorescu, and P. Rodriguez, "Grid-filter design for a multimegawatt medium-voltage voltage-source inverter," IEEE Trans. Ind. Electron., vol. 58, no. 4, pp. 1205-1217, Apr. 2011.

[5] J. Sun, "Impedance-based stability criterion for grid-connected inverters," IEEE Trans. Power Electron., vol. 26, no. 11, pp. 3075-3078, Nov. 2011.
[6] C. M. Wildrick, F. C. Lee, B. H. Cho, and B. Choi, "A method of defining the load impedance specification for a stable distributed power system," IEEE Trans. Power Electron., vol. 10, no. 3, pp. 280-285, May. 1995.

[7] L. Harnefors, M. Bongiorno, and S. Lundberg, "Input-admittance calculation and shaping for controlled voltage-source converters," IEEE Trans. Ind. Electron., vol. 54, no. 6, pp. 3323-3334, Dec. 2007.

[8] L. Xing, F. Feng, and J. Sun, "Optimal damping of emi filter input impedance," IEEE Trans. Ind. Appl., vol. 47, no. 3, pp. 1432-1440, May. 2011.

[9] M. Cespedes and J. Sun, "Adaptive control of grid-connected inverters based on online grid impedance measurements," IEEE Trans. Sustain. Energy, vol. 5, no. 2, pp. 516-523, Apr. 2014.

[10] M. Liserre, G. Buticchi, M. Andresen, G. D. Carne, L. F. Costa, and Z. X. Zou, "The smart transformer: impact on the electric grid and technology challenges," IEEE Ind. Electron. Mag., vol. 10, no. 2, pp. 46-58, Summer 2016.

[11] X. Wang, F. Blaabjerg, M. Liserre, Z. Chen, J. He, and Y. Li, "An active damper for stabilizing power-electronics-based ac systems," IEEE Trans. Power Electron., vol. 29, no. 7, pp. 3318-3329, Jul. 2014.

[12] X.-P. Zhang, C. Rehtanz, and B. Pal, Flexible AC transmission systems: modelling and control. Springer Science \& Business Media, 2012.

[13] M. Liserre, R. Teodorescu, and F. Blaabjerg, "Stability of photovoltaic and wind turbine grid-connected inverters for a large set of grid impedance values," IEEE Trans. Power Electron., vol. 21, no. 1, pp. 263-272, Jan. 2006.

[14] M. M. J. de Vries, M. J. Kransse, M. Liserre, V. G. Monopoli, and J. M. A. Scherpen, "Passivity-based harmonic control through series/parallel damping of an h-bridge rectifier," in IEEE International Symposium on Industrial Electronics, pp. 3385-3390, Jun. 2007.

[15] D. del Puerto-Flores, J. M. A. Scherpen, M. Liserre, M. M. J. de Vries, M. J. Kransse, and V. G. Monopoli, "Passivity-based control by series/parallel damping of single-phase pwm voltage source converter," IEEE Trans. Control Syst. Technol, vol. 22, no. 4, pp. 1310-1322, Jul. 2014.

[16] J. K. Pedersen, F. Blaabjerg, J. W. Jensen, and P. Thogersen, "An ideal pwm-vsi inverter with feedforward and feedback compensation," in Fifth European Conference on Power Electronics and Applications, pp. 501507 vol.5, Sep. 1993.

[17] A. Riccobono and E. Santi, "Positive feedforward control of three-phase voltage source inverter for dc input bus stabilization with experimental validation," IEEE Trans. Ind. Appl, vol. 49, no. 1, pp. 168-177, Jan. 2013.

[18] X. Wang, F. Blaabjerg, and W. Wu, "Modeling and analysis of harmonic stability in an ac power-electronics-based power system," IEEE Trans. Power Electron., vol. 29, no. 12, pp. 6421-6432, Dec. 2014.

[19] Y. W. Li, "Control and resonance damping of voltage-source and currentsource converters with lc filters," IEEE Trans. Ind. Electron., vol. 56, no. 5, pp. 1511-1521, May. 2009.

[20] J. Dannehl, M. Liserre, and F. W. Fuchs, "Filter-based active damping of voltage source converters with lcl filter," IEEE Trans. Ind. Electron., vol. 58, no. 8, pp. 3623-3633, Aug. 2011.

[21] M. Liserre, F. Blaabjerg, and R. Teodorescu, "Grid impedance estimation via excitation of lcl-filter resonance," IEEE Trans. Ind. Appl, vol. 43, no. 5, pp. 1401-1407, Sep. 2007.

[22] R. Pena-Alzola, M. Liserre, F. Blaabjerg, M. Ordonez, and T. Kerekes, "A self-commissioning notch filter for active damping in a three-phase lcl-filter-based grid-tie converter," IEEE Trans. Power Electron., vol. 29, no. 12, pp. 6754-6761, Dec. 2014.

[23] S. G. Parker, B. P. McGrath, and D. G. Holmes, "Regions of active damping control for lcl filters," IEEE Trans. Ind. Appl, vol. 50, no. 1, pp. 424-432, Jan. 2014.

[24] Conseil International des Grands Réseaux Électriques (CIGRÉ). Comité d'Études C6, Benchmark Systems for Network Integration of Renewable and Distributed Energy Resources. CIGRÉ, 2014. 


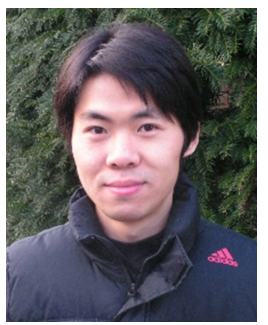

Zhi-Xiang Zou (S'12) received the B.Eng. and $\mathrm{Ph} . \mathrm{D}$. degrees in electrical and engineering from Southeast University, Nanjing, China, in 2007 and 2014, respectively. From 2007 to 2009, he was an engineer in the State Grid Electric Power Research Institute, Nanjing, China. He is currently a scientific staff member at the Chair of Power Electronics, University of Kiel, Germany. His research interests include smart transformers, microgrid stability, and control of grid converters.

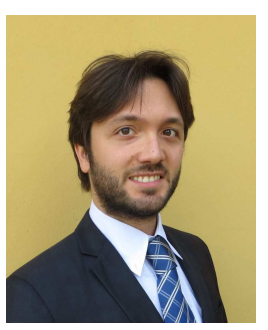

Giampaolo Buticchi (S'10-M'13-SM'17) was born in in Parma, Italy, in 1985 . He received the Masters degree in Electronic Engineering in 2009 and the Ph.D degree in Information Technologies in 2013 from the University of Parma, Italy. In 2012 he was visiting researcher at The University of Nottingham, UK. He is now working as a postdoctoral research associate at the University of Kiel, Germany. His research area is focused on power electronics for renewable energy systems, smart transformer fed micro-grids and reliability in power electronics.

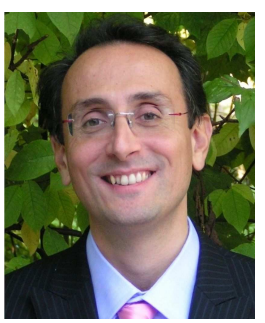

Marco Liserre (S'00-M'02-SM'07-F'13) received the $\mathrm{MSc}$ and $\mathrm{PhD}$ degree in Electrical Engineering from the Bari Polytechnic, respectively in 1998 and 2002. He has been Associate Professor at Bari Polytechnic and Professor at Aalborg University (Denmark). $\mathrm{He}$ is currently Full Professor and he holds the Chair of Power Electronics at ChristianAlbrechts-University of Kiel (Germany). He has published over 300 technical papers (more than 86 of them in international peer-reviewed journals) and a book. These works have received more than 20000 citations. Marco Liserre is listed in ISI Thomson report "The world's most influential scientific minds". He is member of IAS, PELS, PES and IES. He has been serving all these societies in different capacities and he has received several awards. 